\title{
ON RINGS OF INVARIANTS WITH RATIONAL SINGULARITIES
}

\author{
BARBARA R. PESKIN
}

\begin{abstract}
Let $S$ be a noetherian local $k$-algebra and $G$ a finite group of $k$-automorphisms of $S$. If char $k=0$ and $S$ has a rational singularity, then the invariant ring $R=S^{(i}$ does also. However. if char $h \neq 0$, this is rarely true. We examine condition. on wild group actions in dimension two which ensure that the singularity of $R$ is rational. In particular. we develop a criterion in terms of the minimality of $H^{1}((i, S)$.
\end{abstract}

Let $k$ be an algebraically closed field and let $S$ be a local noetherian normal $k$-algebra. The ring $S$ is said to have a rational singularity if for any resolution $f: X \rightarrow \operatorname{Spec} S$, that is, for any proper, birational map $f$ for which $X$ is smooth. the cohomology groups $H^{\prime}\left(X\right.$. " $\left._{X}\right)$ vanish for all $i>0$. Suppose that $G$ is a group of $k$-automorphisms of $S$ such that the ring of invariants $R=S^{(i}$ is also noetherian. Then $R$ is again a normal local ring and we wish to examine conditions on the group action which ensure that $R$ has a rational singularity if $S$ does.

Boutot (unpublished) has proved that this is always the case if $S$ is finitely generated over a field of characteristic zero and $G$ is a linearly reductive group. For finite group actions in characteristic zero, this had been established by Brieskorn [3. Satz 1.7] in dimension two and then generalized to arbitrary dimensions. However, when $k$ has characteristic $p \neq 0$, even finite groups are not linearly reductive if $p$ divides the order of $G$, and. correspondingly, the ring $R$ need not have a rational singularity. Our purpose in this paper is to determine an appropriate extension of Boutot's theorem to finite group actions in characteristic $p$.

If the dimension of $S$ is greater than two. one immediately encounters two difficulties: the proper definition of rational singularity is unclear due to the lack of a resolution theorem for singularities in positive characteristics, and also the invariant ring $R$ need not be Cohen-Macaulay [6, \$1], a necessary condition for rational singularities. Consequently, we confine our attention to the case that $S$ is two-dimensional, where both of these difficulties vanish.

Now for linearly reductive actions in dimension two, Boutot's result is easily extended to characteristic $p$. This is possible because of the generalization to arbitrary characteristic of the Grauert-Riemenschneider vanishing theorem [8. Theorem A]. a key ingredient in Boutot's proof. The hypothesis of linear reductivity

Received by the editors March 29. 1982 and. in revised form. September 9. 1982

1980 Mathematics Subject (lassification. Primary 14L30, 14B05.

Kor words and phrases. Wild group action. rational singularits. 
allows one to realize the invariant ring $R$ as a direct summand of $S$ by means of the Reynolds operator $\rho$ projecting $S$ onto $R$. For finite group actions, the Reynolds operator is given as the normalization $\rho=\operatorname{tr} /|G|$ of the trace map $\operatorname{tr}: S \rightarrow R$, where $|G|$ denotes the order of $G$. Recall that the trace map is defined by $\operatorname{tr}(x)=\Sigma_{\sigma \in G} \sigma x$ for $x \in S$. If $p$ divides $|G|$ - the so-called "wild" actions-the trace map degenerates and linear reductivity is lost. The ring $S$ still maps to $R$ via trace, but the elements of $R$ are annihilated: if $r \in R$, then $\operatorname{tr}(r)=|G| \cdot r=0$.

For the remainder of the paper, we will examine only the "test case" for wild actions, namely the case that $G$ is a cyclic group of order $p^{\nu}$ for some $\nu>0$. We further assume that the induced action of $G$ on $\operatorname{Sec} S$ is free except at the closed point.

To measure the degree to which the trace map deviates from a true projection map we use the cohomology groups $H^{\prime}(G, S)$. For cyclic groups $G$ generated by an element $\sigma$, the groups $H^{i}(G, S)$ are 2-periodic for $i>0$ and have a simple formulation in terms of $\sigma$ and $\operatorname{tr}[7$, p. 141]:

$$
\begin{aligned}
& H^{0}(G, S)=R, \\
& H^{\prime}(G, S)=\{\text { kernel of trace }\} /\{\text { image of }(\sigma-\mathrm{id})\}, \\
& H^{2}(G, S)=R /\{\text { image of trace }\}, \\
& H^{\prime}(G, S)=H^{1^{-2}}(G, S) \text { for } i>2 .
\end{aligned}
$$

Note that for linearly reductive groups $G$, all $H^{i}(G, S)=0$ for $i>0$ by the projection property of trace. For this case $S=R \oplus K$, where $R=S^{G}$ is the image of trace and $K$ is the kernel of trace or, equivalently for linearly reductive groups, the image of $\sigma$ - id. In contrast, for wild group actions we have the following:

LEMMA 1. Let $G$ be a cyclic group of $k$-automorphisms of $S$ such that the action of $G$ on $\operatorname{Spec} S$ is free off of the closed point. If $p$ divides $|G|$, the groups $H^{i}(G, S)$ are nontrivial finite-dimensional vector spaces over $k$ for $i>0$.

Proof. The groups $H^{\prime}(G, S)$ are finite-dimensional $R$-modules. Since the action of $G$ is free off of the closed point of $\operatorname{Spec} S$, the groups are supported at the closed point of $\operatorname{Spec} R$, for $i>0$, hence are actually $k$-vector spaces. Now the map $R \rightarrow S$ is totally ramified at the maximal ideal $\mathrm{m}_{s}$ of $S$ and so $\sigma \equiv$ id $\left(\bmod \mathrm{m}_{S}\right)$. The images of $\sigma-$ id and of $\operatorname{tr}$ are therefore contained in $m_{s}$. Consequently, the groups $H^{\prime}(G, S)$ all contain a copy of $k$ : any element $c \in k$ is fixed by $\sigma$ and cannot lie in the image of $\sigma-$ id nor of tr.

Now suppose that $f: X \rightarrow \operatorname{Spec} R$ is a resolution of the singularity of $R$. We wish to determine when $H^{1}\left(X, \theta_{X}\right)=0$. Let $Y$ be the normalization of $X$ in the fraction field $K(S)$ of $S$ and let $g$ be the map $Y \rightarrow \operatorname{Spec} S$ and $\pi$ the map $Y \rightarrow X$. We then have the diagram of spaces:

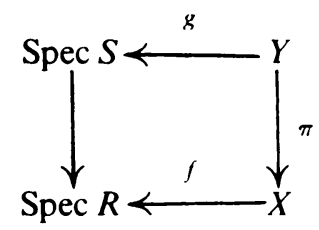


The space $Y$ acquires a $G$-action from the action of $G$ on $K(S)$ and $X$ is precisely the quotient of $Y$ under this action. Although $Y$ may not be a resolution of $\operatorname{Spec} S$. we have

Lemma 2 [5, Proposition 1.2]. Let g: $Y \rightarrow \operatorname{Spec} S$ be a proper, birational map with $Y$ normal. Then if $S$ has a rational singularity, the groups $H^{\prime}\left(Y, \hat{O}_{Y}\right)=0$ for all $i>0$.

Proof. It is enough to check that $H^{\prime}\left(Y, C_{Y}\right)=0$ since the higher cohomology groups vanish by dimension considerations. There exists a normal surface $Z$ and a proper birational map $h: Z \rightarrow Y$ such that $h \circ g: Z \rightarrow \operatorname{Spec} S$ is a resolution of Spec $S$. By the normality of the spaces $h_{*}{ }^{\sigma_{Z}}=\hat{0}_{Y}$. Now $H^{1}\left(Z, c_{Z}\right)=0$ since $S$ has a rational singularity, and so the canonical inclusion $H^{1}\left(Y, h_{*}{ }^{0} Z_{Z}\right) \rightarrow H^{1}\left(Z, 0_{Z}\right) \mathrm{im}-$ plies that $H^{1}\left(Y, \mathrm{O}_{Y}\right)$ is also zero.

Since $H^{i}\left(Y, \theta_{Y}\right)=0$ for all $i>0$, there is a spectral sequence [4. Proposition 5.2.4] relating the group cohomology for $S$ and $\hat{C}_{Y}$ :

$$
H^{p}\left(X, \mathcal{K}^{q}\left(G, 0_{Y}\right)\right) \Rightarrow H^{*}(G, S) \text {. }
$$

where $\mathcal{K}^{q}\left(G, C_{Y}\right)$ is the sheaf whose sections on any affine $U \subset X$ are given by the

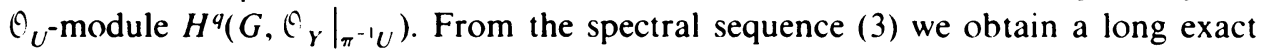
sequence on low-degree terms beginning

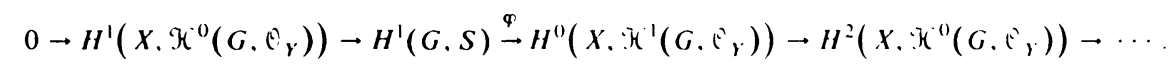

Note that $\mathcal{H}^{0}\left(G, \hat{O}_{Y}\right)$ is equal to $\left({ }^{C_{Y}}\right)^{G}=\mathfrak{C}_{X}$ so that the last term in (4) is zero and hence the map $\varphi$ is surjective.

Proposirion 5. Suppose that $S$ has a rational singularity. Then $R$ has a rational singularity if and only if the map

$$
\varphi: H^{1}(G, S) \rightarrow H^{0}\left(X, \mathbb{R}^{-1}\left(G, \mathbb{C}_{Y}\right)\right)
$$

is injective.

Proof. This is an immediate consequence of the sequence (4). The sheaf $H^{1}\left(X, \mathcal{H}^{0}\left(G, \mathcal{O}_{Y}\right)\right)$ is equal to $H^{1}\left(X, \vartheta_{X}\right)$, so the vanishing of $H^{1}\left(X, \bullet_{X}\right)$ is equivalent to the injectivity of $\varphi$.

We remark that for linearly reductive actions $\varphi$ is trivially injective since $H^{\prime}(G, S)$ and $\mathcal{H}^{i}\left(G, \theta_{Y}\right)$ are zero, as noted above. Hence $R$ always has rational singularities. For wild actions, this is rarely true. However, there is one case in which the injectivity of $\varphi$ is guaranteed.

THEOREM 6. Let $G$ be a cyclic group of order $p^{\nu}$ which acts freely off of the closed point of $\operatorname{Spec} S$. If $H^{\prime}(G, S)=k$ and $S$ has a rational singularity, then $R$ has a rational singularity.

Proof. The vector space $H^{1}(G, S)$ contains the constants $k$ as shown in Lemma 1 and so, by hypothesis, contains only the constants. In a similar fashion, we show that $\mathcal{H}^{1}\left(G, \mathcal{O}_{Y}\right)$ also contains the constants as global sections. Let $Z \subset Y$ denote the ramification locus of $\pi: Y \rightarrow X$ and $\pi(Z)$ its image in $X$. Note that $Z$ is nontrivial since $X$ is smooth and that $\pi(Z)$, the support of $\mathcal{H}^{1}\left(G, \Theta_{Y}\right)$, is contained in the exceptional locus of $X$. Now the image of $\sigma-$ id lies in $\mathscr{G}(Z)$, the ideal sheaf of $Z$, 
since $\pi$ is wildly ramified there, and so the constants, which are fixed by $\sigma$, cannot lie in $\operatorname{im}(\sigma-$ id $)$. They therefore determine nonzero classes in $\mathrm{K}^{\prime}\left(G, C^{\prime}\right)$ and it is easy to check that $\varphi$ maps the constants in $H^{\prime}(G, S)$ to the constants in $H^{\prime \prime}\left(X, H^{\prime}\left(G, O_{\gamma}\right)\right)$, giving the desired injection.

Consequently, if $H^{\prime}(G, S)$ is minimal for wild group actions, the singularity of $R$ is rational.

Even though this criterion seems unnecessarily strong to guarantee that $\varphi$ is injective, in many families of actions it is precisely when $H^{\prime}(G, S)=k$ that $R$ has a rational singularity, as the following example illustrates.

EXAmPle 7. Let $k$ be a field of characteristic 3 and let $S=k \llbracket u, v \rrbracket$. Suppose $G=\mathbf{Z} / 3$ and $\sigma$ is a generator of $G$. The action of $\sigma$ is defined by two power series, the images of $u$ and $v$ under $\sigma$. If the linear terms for the action determine a transformation with a single Jordan block then coordinates for $S$ can be chosen so that $\sigma$ has the form

$$
\sigma u=u+y^{\prime}, \quad \sigma v=v+u
$$

where

$$
y=v \cdot \sigma v \cdot \sigma^{2} v=v(v+u)\left(v+2 u+y^{\prime}\right)
$$

for some $i>0[6,4.12$ and 5.15]. Note that $v$ is invariant under $\sigma$ and that equation (8) may be solved recursively for $y$ in terms of $u$ and $v$ alone.

The invariant ring $R$ is generated by $y$ and the two additional elements

$$
x=u \cdot \sigma u \cdot \sigma^{2} u, \quad z=u^{2}+y^{\prime} v+2 y^{\prime} u,
$$

subject to the single relation

$$
z^{3}+y^{2 \imath} z^{2}=y^{3 \imath+1}+x^{2} .
$$

A straightforward calculation now confirms that $H^{1}(G, S)=k$ if and only if $i=1$, the only case in which equation (9) defines a rational singularity. (The reader may consult Artin's list of rational double points in characteristic $p$ [2] to verify rationality. For $i=1$, the singularity is a rational double point of type $E_{6}$.)

It is interesting to note that this is also the only case in which $H^{2}(G, S)=k$, that is, in which the image of trace covers the maximal ideal $\mathrm{m}_{R}$ of $R$. In general the image of trace is given by the $\mathrm{m}_{R}$-primary ideal $\left(x, y^{i}, z\right)$ and both $H^{1}(G, S)$ and $H^{2}(G, S)$ are generated by the elements $y^{j}$ for $0 \leqslant j \leqslant i-1$.

Despite the abundance of examples like the one above, the next example shows that the condition $H^{\prime}(G, S)=k$ is indeed too strong.

EXAMPLE 10 . Let $k$ be a field of characteristic 2 and consider the $\mathbf{Z} / 2$-action on $S=k \llbracket u, v \rrbracket$ generated by the automorphism

$$
\sigma u=u+y^{i}, \quad \sigma v=v+x
$$

where

$$
\begin{aligned}
& x=u \cdot \sigma u=u\left(u+y^{i}\right), \\
& y=v \cdot \sigma v=v(v+x)
\end{aligned}
$$


Again $x$ and $y$ are invariant under $\sigma$ and the equations (11) can be solved recursively for $x$ and $y$ in terms of $u$ and $v$. The ring of invariants in this case is generated by the three elements $x, y$, and $z=x u+y^{\prime} v$, subject to the relation

$$
z^{2}+x y^{\prime} z+x^{3}+y^{21+1}=0
$$

(see [1]).

If $i=1$, then $H^{1}(G, S)=k$ and the resulting singularity is a rational double point of type $D_{4}$. However, the singularity is also a rational double point (of type $E_{8}$ ) if $i=2$ and in this case $H^{1}(G, S)$ is 2-dimensional. For $i>2$. the singularity is no longer rational. Note that for $\mathbf{Z} / 2$-actions in characteristic 2 , the operators trace and $\sigma-$ id are the same and so, as above, $H^{1}=H^{2}$. The image of trace is again the $\mathrm{m}_{R}$-primary ideal $\left(x, y^{\prime}, z\right)$ and $H^{\prime}(G, S)$ is generated by the $y^{\prime}$ for $j<i$.

Of course the invariant ring need not define a hypersurface singularity. We now consider an action leading to a surface in 4-space.

EXAMPLE 13. As in Example 7, let $k$ be a field of characteristic 3 and $S=k \llbracket u, v \rrbracket$. Consider the following $\mathbf{Z} / 3$-action with generator $\sigma$ having two Jordan blocks:

$$
\begin{aligned}
& \sigma u=\frac{u}{1-u}=u+u^{2}+u^{3}+\cdots . \\
& \sigma v=\frac{v}{1-v}=v+v^{2}+v^{3}+\cdots .
\end{aligned}
$$

The invariant ring $R$ is generated by the four elements

$$
\begin{aligned}
x & =u \cdot \sigma u \cdot \sigma^{2} u, \\
y & =v \cdot \sigma v \cdot \sigma^{2} v, \\
z & =y u(u+x)-x v(v+y), \\
w & =y(u-x)+x(v-y)+u v(u+x)(v+y) .
\end{aligned}
$$

The relations among the generators are given by the $2 \times 2$-minors of the $2 \times 3$ matrix

$$
\left(\begin{array}{ccc}
x & x y & z \\
y-x & z & u+x y
\end{array}\right) .
$$

A rather tedious calculation confirms that $H^{\prime}(G, S)=k$ and so the singularity is a rational triple point. Again we note that the invariants (14) all lie in the image of trace, hence $H^{2}(G, S)$ is also $k$.

Finally, we pose the following question: In all of the above examples $H^{1}(G, S)=$ $H^{2}(G, S)$; is this generally the case for wild actions? This would be an extremely useful result, as the group $H^{2}(G, S)$ is often easier to compute than $H^{1}(G, S)$ and has a simpler interpretation in terms of the degeneracy of the trace map.

\section{REFERENCES}

I. M. Artin. W'ildly ramified Z/2 actions in dimension two. Proc. Amer. Math. Sox. 52 (1975). 60)-64.

2. Conerings of the rational double points in characteristic $p$, in Complex Analysis and Algchraic (icometry, Cambridge Univ. Press, Cambridge. 1977

3. E. Brieskorn, Rationale Singularitäten komplexer Flächen. Invent. Math. 4 (1968). 336-358.

4. A. Grothendieck. Sur quelques points d'algèbre homologique'. Tôhoku Math. J. 9 (1957), 119-221 
5. J. Lipman. Rational singularities with applications to algebraic surfaces and unique factorization. Publ. Math. Inst. Hautes Études Sci. 36 (1969), 195-280.

6. B. R. Peskin, Quotient-singularities and wild p-cyclic actions. J. Algebra (to appear).

7. J.-P. Serre. Corps locaux, Hermann, Paris, 1962.

8. J. M. Wahl, Vanishing theorems for resolutions of surface singularities, Invent. Math. 31 (1975), 17-41.

Department of Mathematics, University of Illinois at Urbana-Champaign, Urbana. Illinois 61801

Current address: Department of Mathematics. Mount Holyoke College. South Hadley. Massachusetts 01075 